\title{
CARACTERIZACIÓN DEL PROCESO DE ADSORCIÓN DE 3-CLORO FENOL DESDE SOLUCIÓN ACUOSA SOBRE CARBON ACTIVADO POR CALORIMETRIA DE INMERSIÓN
}

\author{
Liliana Giraldo \\ Departamento de Química, Facultad de Ciencias, Universidad Nacional de Colombia, Carrera 30 No 45-03, Bogotá, Colombia \\ Juan Carlos Moreno* \\ Departamento de Química, Facultad de Ciencias, Universidad de los Andes, Carrera 1 No 18 A-10, Bogotá, Colombia
}

Recebido em 11/8/08; aceito em 16/3/09; publicado na web em 10/8/09

\begin{abstract}
CHARACTERIZATION OF 3-CHLOROPHENOL ADSORPTION PROCESS FROM AQUEOUS SOLUTION ON ACTIVATED CARBON BY IMMERSION CALORIMETRY. The immersion enthalpy of activated carbon in 3-chlorophenol solutions, of 100 $\mathrm{mg} \mathrm{L}^{-1}$, is determined at different $\mathrm{pH}$ values between 3 and 11 with results between 37.6 and $21.2 \mathrm{~J} \mathrm{~g}^{-1}$. The 3-chlorophenol adsorbed quantities on the activated carbon during the calorimetric experience, are between 1.13 and $2.19 \mathrm{mg} \mathrm{g}^{-1}$, for different $\mathrm{pH}$ values of the solution. The 3-chlorophenol adsorbed quantity and the immersion enthalpy decrease by increasing of the $\mathrm{pH}$ solution, while increasing the adsorbed quantity increases the immersion enthalpy value.
\end{abstract}

Keywords: activated charcoal; enthalpy immersion; 3-chloro phenol.

\section{INTRODUCCION}

La adsorción de fenoles sobre carbones activados depende tanto de las características físicas del sólido como área superficial y porosidad como de la superficie química. ${ }^{1}$ Los estudios acerca del proceso de adsorción sobre carbón activado de fenol y sus compuestos derivados presentes en soluciones acuosas se han realizado desde hace tiempo y se continúan actualmente $\mathrm{e}^{2,3}$ mostrando que dicho proceso presenta algunas complejidades y dependencias con las características de la solución, como $\mathrm{pH}$, fuerza iónica, grado de dilución entre otras, que a su vez influyen en el mecanismo de adsorción de los compuestos fenólicos. ${ }^{4}$

La calorimetría de inmersión se usa para conocer, dependiendo de las condiciones termodinámicas del sistema, el calor que se produce cuando se ponen en contacto un sólido y un líquido y por tanto la entalpía de inmersión, del proceso de contacto, que se establece para una cantidad específica del sólido que se estudia. ${ }^{5}$

La intensidad de la interacción entre la solución y el carbón activado se puede determinar por medio de la entalpía de inmersión del sólido en soluciones que contengan sustancias activas con la superficie y que permiten relacionar las propiedades fisicoquímicas del carbón activado con la capacidad de adsorción de los compuestos que están en solución. ${ }^{6-9}$

La adsorción en fase líquida es un proceso más complicado que la adsorción en fase gaseosa o vapor. ${ }^{10}$ Las principales diferencias entre la adsorción en fase acuosa y en fase gaseosa son las siguientes. ${ }^{11}$ Primero, la adsorción en fase acuosa es esencialmente un proceso de intercambio, y muchas moléculas se adsorben no sólo porque son atraídas por el sólido, sino también porque la solución puede rechazarlas; un ejemplo de esto es la adsorción de moléculas hidrófobas por adsorbentes hidrófobos en solución acuosa. En este caso la adsorción se debe, fundamentalmente, al rechazo del agua hacia las moléculas hidrófobas y no la atracción de éstas por la superficie del sólido. Segundo, las isotermas en solución pueden presentar no-idealidad no solo debido a las interacciones laterales entre moléculas adsorbidas, si no también, debido a no-idealidad en solución. Tercero, la adsorción en multicapa es menos corriente

*e-mail: jumoreno@uniandes.edu.co en fase acuosa que en fase gaseosa, debido a las mayores fuerzas de interacción apantallantes en fluidos condensados.

Cada uno de los aspectos que se mencionan anteriormente, están presente cuando se realiza la medida calorimétrica y por tanto contribuyen en la magnitud total de calor que se determina cuando se ponen en contacto el sólido y la solución; las interacciones que se producen entre éstos son: interacción entre el agua, y los grupos oxigenados de la superficie del carbón activado, el llenado de los poros y la adsorción en la superficie ${ }^{12}$ y por otro lado la adsorción y las interacciones con el soluto que por disociarse en solución dependerán de las condiciones de la misma. ${ }^{13}$

En este trabajo se estudia la intensidad de la adsorción de 3-cloro fenol desde soluciones acuosas sobre un carbón activado preparado a partir de cuesco de palma africana, por medio de la determinación de isotermas de adsorción y de entalpías de inmersión a diferentes valores de $\mathrm{pH}$, que se varían entre 3 y 11 . Se determinan las cantidades adsorbidas de 3-cloro fenol sobre el carbón activado durante la experiencia calorimétrica, y se establecen relaciones entre las cantidades adsorbidas y los valores entálpicos de la interacción sólido-líquido.

\section{PARTE EXPERIMENTAL}

\section{Caracterización del carbón activado}

El carbón activado que se emplea en este trabajo se obtiene por activación física de cuesco de palma africana. Inicialmente se lleva a cabo una carbonización del material particulado a $723 \mathrm{~K}$ en atmósfera de $\mathrm{N}_{2}$ y posteriormente se realiza la activación con $\mathrm{CO}_{2}$ a $1073 \mathrm{~K}$ durante $2 \mathrm{~h}$. Una vez obtenido el carbón activado se caracteriza mediante la isoterma de adsorción de $\mathrm{N}_{2}$ a $77 \mathrm{~K}$ en un equipo convencional volumétrico, Autosorb 3B, Quantachrome.

Los contenidos de grupos ácidos y básicos totales se determinan por el método de titulación de Boehm, ${ }^{14,15}$ para lo cual se equilibran muestras de carbón activado en soluciones ácida y básica y posteriormente se titula con una solución estándar de hidróxido de sodio o ácido clorhídrico según sea el caso, midiendo durante la titulación el pH de la solución con un pH meter CG 840B Schott.

El pH en el punto de carga cero, indica la carga total del sólido, y se determina por el método de titulación de masas, ${ }^{6}$ para su determina- 
ción se pesan cantidades del carbón activado en un rango de 0.010 a $0.600 \mathrm{~g}$, se colocan en frascos de vidrio de $50.0 \mathrm{~mL}$ y se agrega 10.0 $\mathrm{mL}$ solución de cloruro de sodio, $\mathrm{NaCl}, 0.1 \mathrm{M}$. Los frascos se tapan y se dejan en agitación a temperatura de $298 \mathrm{~K}$ durante 48 h, para que las cargas del carbón se equilibren. Pasadas las 48 h se mide el pH de cada una de las soluciones con un $\mathrm{pH}$ meter CG 840B Schott.

\section{Determinación de isotermas de adsorción de 3-cloro fenol a diferente $\mathbf{p H}$}

En la Tabla 1 se presentan algunas de las propiedades del 3-cloro fenol que es el adsorbente orgánico que se estudia desde solución acuosa. ${ }^{16}$ Los cloro fenoles son compuestos orgánicos que aparecen con frecuencia en las aguas residuales y superficiales ya sea como desecho industrial o formados por la cloración de las aguas; dada su alta toxicidad se retiran de las aguas por medio de adsorción. ${ }^{2}$

Tabla 1. Propiedades del 3-cloro fenol

\begin{tabular}{lccc}
\hline $\begin{array}{l}\text { Peso molecular } \\
\left(\mathrm{mol} \mathrm{g}^{-1}\right)\end{array}$ & $\begin{array}{c}\text { Longitud de } \\
\text { onda de máxima } \\
\text { absorción } \lambda\end{array}$ & pKa a $298 \mathrm{~K}$ & $\begin{array}{c}\text { Solubilidad } \\
\left(\mathrm{g} / 100 \mathrm{~g} \mathrm{H}_{2} \mathrm{O}\right)\end{array}$ \\
\hline 129 & 273 & 8.8 & 2.6 \\
\hline
\end{tabular}

Para determinar la cantidad que adsorbe el carbón activado de 3-cloro fenol se colocan $0,5 \mathrm{~g}$ de éste en frascos de vidrio y $250 \mathrm{~mL}$ de las respectivas soluciones acuosas de 3-cloro fenol en un rango de concentración entre 20 a $120 \mathrm{mg} \mathrm{L}^{-1}$. Las muestras se agitan mecánicamente y se mantienen a una temperatura de $298 \mathrm{~K}$, por un periodo de $48 \mathrm{~h}$ que asegura el equilibrio del sistema, durante este tiempo el pH de la solución se mantiene en un valor fijo, en un rango entre 3 y 11, mediante la adición de soluciones diluidas de $\mathrm{HCl}$ o de $\mathrm{NaOH}$. La concentración de equilibrio del compuesto fenólico en las soluciones después de la adsorción se determina, previa calibración a cada valor de $\mathrm{pH}$ a la longitud de onda de máxima absorción, con un equipo espectrofotométrico uv-vis Milton Roy Co. Spectronic Genesys SN.

\section{Determinación de la cantidad de 3-cloro fenol adsorbido en la calorimetría}

La concentración en las soluciones del 3-cloro fenol, una vez se lleva a cabo la calorimetría de inmersión, se determina previa calibración a cada valor de $\mathrm{pH}$ a la longitud de onda de máxima absorción, con un equipo espectrofotométrico uv-vis Milton Roy Co. Spectronic Genesys SN.

\section{Determinación de la entalpía de inmersión}

Se determinan las entalpías de inmersión del carbón activado en soluciones de 3-cloro fenol de $100 \mathrm{mg} \mathrm{L}^{-1}$ a los valores de $\mathrm{pH}$ escogidos. Para dicha determinación se usa un microcalorímetro de conducción de calor, que se muestra en la fotografía de la Figura $1 \mathrm{~S}$, con una celda calorimétrica en acero inoxidable. ${ }^{13}$ Se colocan en la celda $30 \mathrm{~mL}$ de la solución a utilizar, que se ha mantenido en un termostato a $298 \mathrm{~K}$; se pesa una muestra de carbón activado del orden de $0.500 \mathrm{~g}$ y se coloca dentro de la celda calorimétrica en una ampolleta de vidrio, se ensambla el microcalorímetro. Cuando el equipo alcanza una temperatura de alrededor de $298 \mathrm{~K}$, se inicia el registro de potencial de salida por un periodo de aproximadamente 15 min tomando lecturas de potencial cada $20 \mathrm{~s}$, se procede a realizar el rompimiento de la ampolleta de vidrio, se registra el efecto térmico generado y se continua con las lecturas de potencial por aproximada- mente 15 min más, una vez transcurrido dicho tiempo se suministra a la celda una cantidad trabajo eléctrico conocido, entre 0.5 y 1.0 $\mathrm{J}$ a través de la resistencia de calentamiento, y se continúa con las lecturas de potencial hasta que éstas sean nuevamente constantes, y se determina la concentración de la solución del 3-cloro fenol.

\section{RESULTADOS Y DISCUSIÓN}

Como se ha dicho el proceso de adsorción depende de las características del adsorbente y por esta razón se determinan el valor del área superficial del carbón activado, que se obtiene a partir de cuesco de palma africana, que es de $685 \mathrm{~m}^{2} \mathrm{~g}^{-1}$, que está de acuerdo con valores obtenidos en otros trabajos, ${ }^{17}$ en los que se presentan condiciones similares en su preparación. El volumen de microporos es de $0.33 \mathrm{~cm}^{3} \mathrm{~g}^{-1}$, que exhibe un contenido de microporosidad de alrededor de $78 \%$. El contenido de grupos ácidos y básicos totales es de 0.96 y $0.38 \mathrm{mmol} \mathrm{g}^{-1}$ respectivamente lo que hace que el $\mathrm{pH}$ en el punto de carga cero, $\mathrm{pH}_{\mathrm{PZC}}$, sea ligeramente ácido, con un valor de 6.8, que se debe tener en cuenta para la adsorción del 3-cloro fenol a los diferentes valores de $\mathrm{pH}$, dado que éste modifica la carga superficial del carbón activado.

Buena información sobre el proceso de adsorción de un compuesto en solución sobre un carbón activado se obtiene a partir de las isotermas de adsorción dado que éstas representan estados de equilibrio del sistema y suministran información sobre la capacidad de adsorción a unas determinadas condiciones, en este estudio se observa la influencia del pH en la adsorción del 3-cloro fenol sobre un carbón activado con un contenido de grupos ácidos mayor que el contenido de grupos básicos y que para los valores de pH 3 y 5 estará cargado positivamente, al pH 7 muy cercano al $\mathrm{pH}_{\mathrm{PZC}}$ de 6.8 tendrá un balance de carga y para los pH de 9 y 11 estará cargado negativamente. Las características de carga del sólido y del soluto afectan la adsorción y esto se refleja en las isotermas de adsorción que se presentan en la Figura 1; en ésta se observa que a los $\mathrm{pH}$ de 5 y 3 se obtienen las mayores cantidades adsorbidas, siendo un $2.6 \%$ mayor a $\mathrm{pH} 5$ que es el pH de máxima adsorción. La adsorción disminuye a pH 7 aunque aún es considerable y la menor adsorción de 3-cloro fenol se obtiene a los pH 9 y 11 con valores de 73.6 y 46.4 $\mathrm{mg} \mathrm{g}^{-1}$ respectivamente.

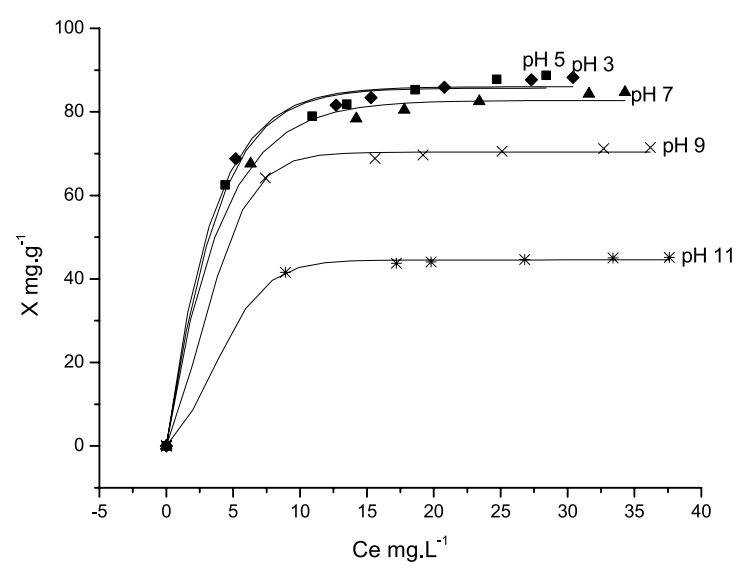

Figura 1. Isotermas de adsorción de 3-cloro fenol a diferentes valores de pH y $298 K$

Los valores de las entalpías de inmersión se evalúan a partir de termogramas de potencial termométrico en función del tiempo, en donde el calor desprendido en el proceso de adsorción es proporcional al área bajo la curva del pico que se genera por el efecto térmico. En la Figura 2 se muestran termogramas típicos para la inmersión del 
carbón activado en solución de 3-cloro fenol de $100 \mathrm{mg} \mathrm{L}^{-1}$ a pH de 5 y de 11 que corresponden a los valores de $\mathrm{pH}$ en los que se presenta la mayor y menor interacción al poner en contacto el sólido y el líquido, y que como se aprecia en la Figura 2, las áreas bajo la curva de los picos son diferentes y que los efectos que se producen a cada uno de los valores de $\mathrm{pH}$ son bien distinguibles.

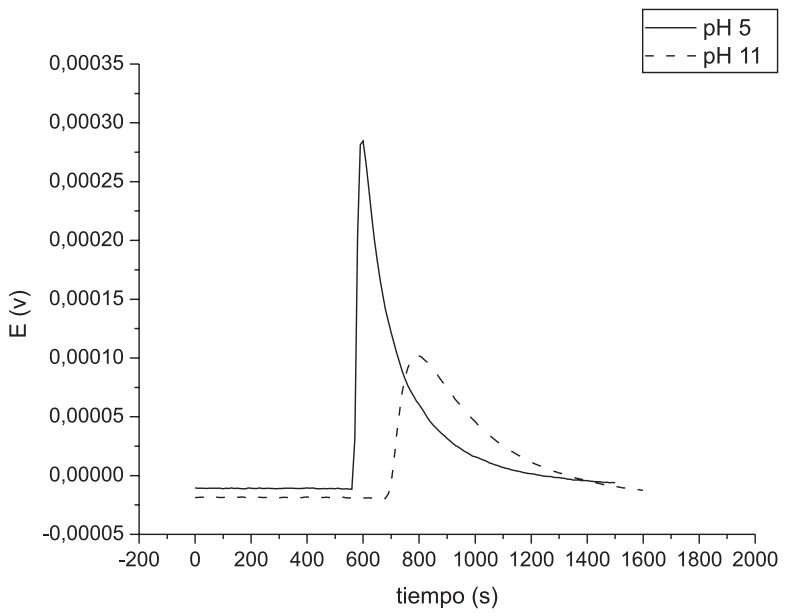

Figura 2. Termogramas de la inmersión del carbón activado en solución de 3-cloro fenol de $100 \mathrm{mg} \mathrm{L}^{-1}$ a los $\mathrm{pH}$ de 5 y 11. T $298 \mathrm{~K}$

En la Figura 3 se presentan la relación entre la entalpía de inmersión del carbón activado en soluciones del fenol a los diferentes valores de $\mathrm{pH}$ y se observa que para $\mathrm{pHs}$ de 3 y 5 los valores para la entalpía de inmersión son cercanos, aunque es mayor para el pH 5, de $37.6 \mathrm{~J}$ $\mathrm{g}^{-1}$, en el cual se presenta la mayor interacción y la mayor cantidad de 3-cloro fenol adsorbido en las condiciones experimentales del trabajo, para pH superiores la entalpía de inmersión disminuye y se presenta el menor valor para la entalpía de inmersión al $\mathrm{pH} 11$, de $21.2 \mathrm{~J} \mathrm{~g}^{-1}$. De acuerdo con el valor obtenido para el $\mathrm{pH}_{\mathrm{PZC}}$ para el carbón activado de 6.8 , a valores de $\mathrm{pH}$ menores a éste la superficie del sólido tendrá una carga positiva y el 3-cloro fenol con un valor de pKa de 8.8 a estos valores de $\mathrm{pH}$ estará sin disociar y la adsorción se lleva a cabo porque predominan las fuerzas de dispersión, en tanto que a valores de $\mathrm{pH}$ mayores la adsorción del fenol disminuye porque se presentan fuerzas electrostáticas repulsivas que predominan en el sistema.

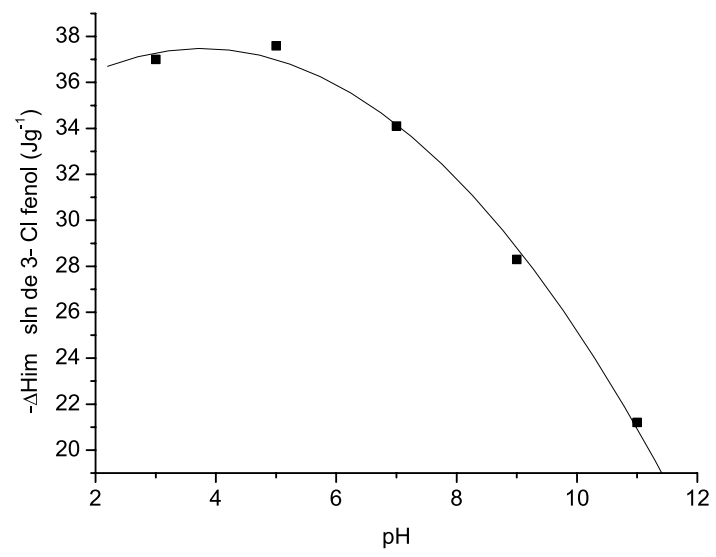

Figura 3. Entalpías de inmersión en función del pH. T $298 \mathrm{~K}$

En la Figura 4, que presenta un comportamiento similar a la Figura 3, se muestra la cantidad adsorbida de 3-cloro fenol durante el tiempo en que se lleva a cabo la experiencia calorimétrica. La cantidad adsorbida del soluto en estas condiciones es proporcional a la cantidad de calor que se genera en el sistema cuando se ponen en contacto el sólido y el líquido y que corresponde a la suma de todas las interacciones tanto físicas como químicas que se llevan a cabo, en las que la presencia de agua como solvente tiene una marcada influencia. Nuevamente se observa que al disminuir el valor del $\mathrm{pH}$ la interacción disminuye y las cantidades adsorbidas de 3-cloro fenol sobre el carbón activado son menores; para los valores de $\mathrm{pH}$ de 3 y 5 la cantidad adsorbida del soluto es la misma, de $2.19 \mathrm{mg} \mathrm{g}^{-1}$, y para valores de $\mathrm{pH}$ menores dicha cantidad disminuye hasta tener el menor valor a $\mathrm{pH} 11 \mathrm{de} 1.13 \mathrm{mg} \mathrm{g}^{-1}$.

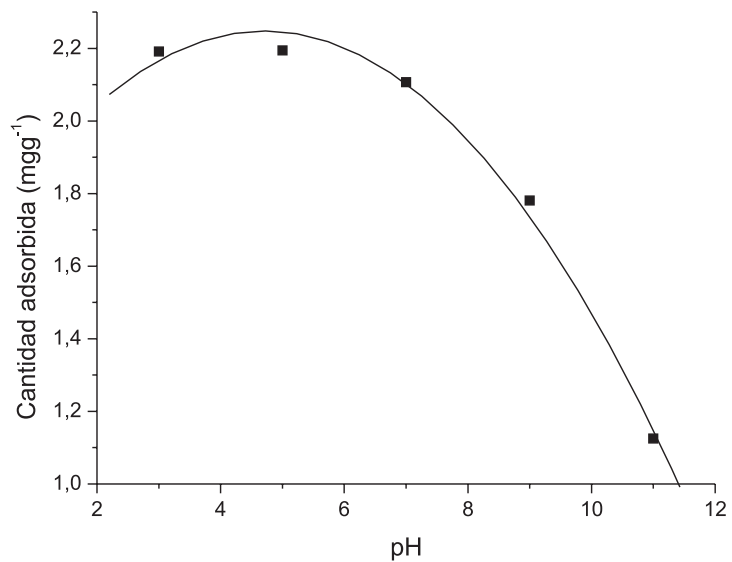

Figura 4. Cantidad de 3-cloro fenol adsorbida sobre el carbón activado en función del pH. T $298 \mathrm{~K}$

La Figura 5 presenta la relación entre la cantidad adsorbida de 3-cloro fenol, después de realizar la calorimetría, y la entalpía de inmersión. Se aprecia que al aumentar la cantidad adsorbida del fenol sobre el carbón activado, los valores de la entalpía de inmersión también aumentan; la relación presenta un comportamiento de segundo orden que indica que las interacciones entre el sólido y la solución no son simples, ya que se ven afectadas por las condiciones electrostáticas en que se encuentra el carbón activado en cada valor de pH y la distribución de las especies del soluto, que se modifica también con esta condición y que favorece o desfavorece las fuerzas que intervienen en la adsorción del soluto sobre el carbón activado. La entalpía de inmersión del carbón activado en soluciones acuosas de 3-cloro fenol, indica la energía que se involucra en la interacción entre el sólido y la solución, que produce la retención del soluto y por tanto esta propiedad permite establecer como influye el $\mathrm{pH}$ en el proceso de adsorción y se constituye en una característica del sistema. ${ }^{18,19}$

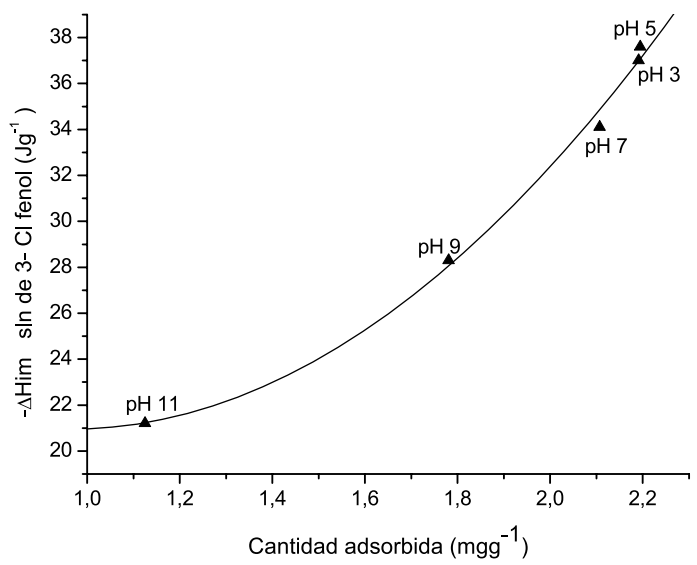

Figura 5. Relación entre la entalpía de inmersión del carbón activado en solución de 3-cloro fenol de $100 \mathrm{mg} \mathrm{L}^{-1}$ y la cantidad adsorbida a $298 \mathrm{~K}$ 
La cantidad adsorbida de 3-cloro fenol en el carbón activado se establece al conocer la concentración de éste en la solución inicial, Ce, y final, Cf , una vez se ha llevado a cabo la adsorción, por lo que dichas concentraciones proporcionan información del proceso y se relacionan con otras propiedades de caracterización, ${ }^{20}$ generalmente con la cantidad retenida por el sólido para obtener las isotermas de adsorción y con los estados de inicial y final del sistema una vez ocurre la adsorción y que definen la energía libre de dicho proceso. En la Figura 6, se muestra la relación entre $\mathrm{Cf} / \mathrm{Ce}$, y la entalpía de inmersión del carbón activado para los valores de $\mathrm{pH}$ considerados, los menores valores para la relación de concentraciones se obtienen para las mayores adsorciones del 3-cloro fenol y a su vez éstas presentan los valores más altos para la entalpía de inmersión; se obtiene una función de segundo orden con un coeficiente de correlación de 0.995 , que indica la favorabilidad del proceso de adsorción en los $\mathrm{pH}$ menores.

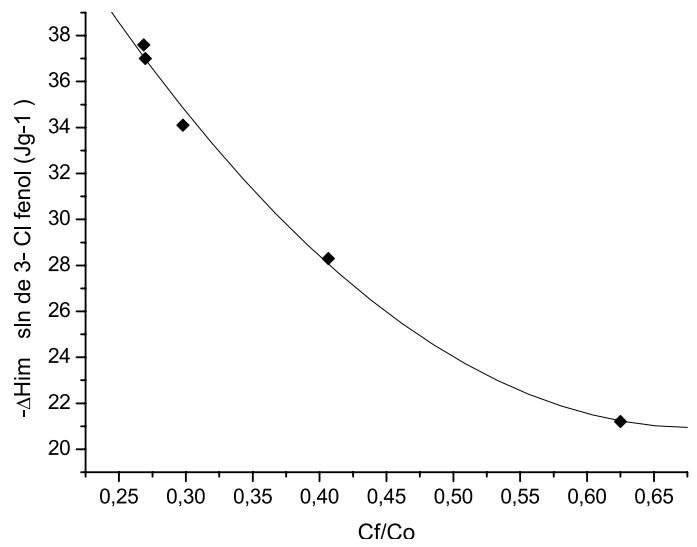

Figura 6. Entalpías de inmersión en función de la relación de concentraciones inicial y final, $\mathrm{Cf} / \mathrm{Co}$

Finalmente en la Figura 7 se muestran la entalpía de inmersión y la cantidad adsorbida en función de la concentración final de la solución, Cf, se aprecia que las dos variables de caracterización presentan un comportamiento lineal con respecto a la concentración del 3-cloro fenol una vez se realiza la calorimetría, indicando que a medida que aumenta el contenido de soluto en la solución final, la cantidad adsorbida y la entalpía de inmersión disminuyen, comportamiento que está de acuerdo con el que se presentó anteriormente en la Figura 6. Estos resultados son comparables con los que obtienen Villacañas y colaboradores, ${ }^{21}$ quienes estudian la adsorción de compuestos orgánicos simples sobre cuatro carbones activados a $\mathrm{pH}$ ácido y básico y encuentran que la adsorción de estos compuestos es mayor a $\mathrm{pH}$ bajos, pero la adsorción depende no solo del $\mathrm{pH}$ de la solución sino también de la química superficial del carbón. La entalpía de inmersión refleja lo que ocurre en el sistema y muestra como para concentraciones finales similares, los valores de entalpía son coincidentes y además pone de manifiesto las interacciones del agua con el sólido que hacen que las pendientes de las líneas, que se muestran en la Figura 7, sean diferentes.

\section{CONCLUSIONES}

La cantidad adsorbida y la entalpía de inmersión de 3-cloro fenol desde solución acuosa sobre un carbón activado preparado a partir de cuesco de palma africana, con un valor de área superficial de $685 \mathrm{~m}^{2}$ $\mathrm{g}^{-1} \mathrm{y}$ con un $\mathrm{pH}$ en el punto de carga cero de 6.8, en función del $\mathrm{pH}$ de la solución muestran que éstas disminuyen con el aumento del $\mathrm{pH}$.

Las entalpías de inmersión del carbón activado en soluciones de 100 $\mathrm{mg} \mathrm{L}^{-1}$ de 3-cloro fenol a los diferentes valores de $\mathrm{pH}$ se encuentran en- tre 37.6 y $21.2 \mathrm{~J} \mathrm{~g}^{-1}$, e indican que el pH de mayor interacción entálpica es 5 , en acuerdo con los valores de la capacidad de adsorción.

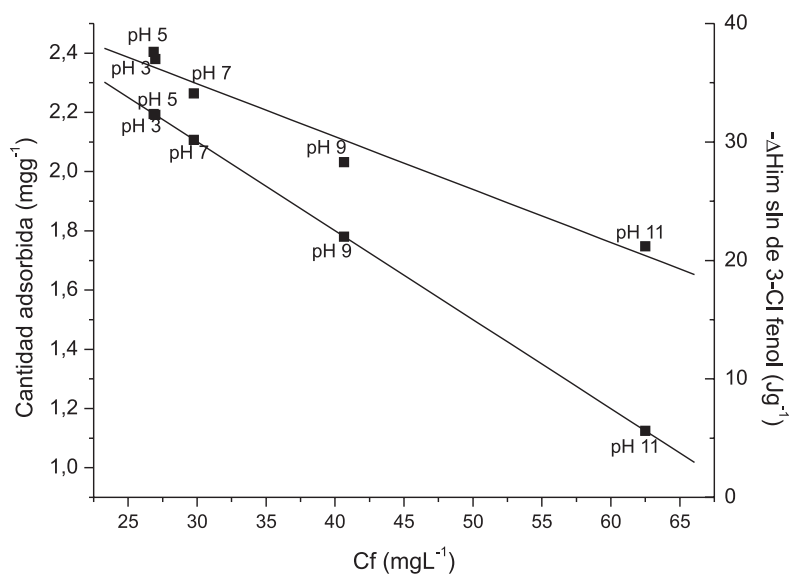

Figura 7. Entalpía de inmersión de carbón activado en solución de $100 \mathrm{mg}$ $L^{-1}$ de 3-cloro fenol y cantidad adsorbida del soluto en función de la concentración final Cf a $298 \mathrm{~K}$

Los valores para las entalpías de inmersión son mayores a $\mathrm{pH}$ ácidos, en los que el soluto se encuentra en su forma asociada y la adsorción se establece mediante fuerzas dispersivas en tanto que a $\mathrm{pH}$ 11 se encuentra el menor valor para la entalpía de inmersión debido a la repulsión electrostática entre el soluto disociado y la superficie del carbón activado cargada negativamente.

\section{MATERIAL SUPLEMENTARIO}

La Figura 1S está disponible en http://quimicanova.sbq.org.br, en arquivo PDF, con acceso libre.

\section{AGRADECIMIENTOS}

Al Convenio Marco entre la Universidad de los Andes y la Universidad Nacional de Colombia y al Acta de Acuerdo entre los Departamentos de Química de las dos Universidades.

\section{REFERENCIAS}

1. McGuire, M. J.; Suffet, I. H.; Treatment of water by granular activated carbon, American Chemical Society: Washington D.C., 1983, p. 29.

2. Wang, S. L.; Tzou, Y. M.; Lu, Y. H.; Sheng, G.; J. Hazard. Mater. 2007, 147, 313 .

3. Merzougui, Z.; Addoun, F.; Desalination 2008, 222, 394.

4. Chien-To, H.; Hsisheng, T.; J. Colloid Interface Sci. 2000, 230, 171.

5. Moreno, J. C.; Giraldo, L. En Sólidos porosos. Preparación, caracterización y aplicaciones; Moreno, J. C., ed.; Uniandes: Bogotá, 2007, p. 281.

6. Giraldo, L.; Moreno, J. C.; Rev. Colomb. Quim. 2003, 32, 45.

7. Lopez-Ramon, M.; Stoeckli, F.; Moreno-Castilla, C.; Carrasco-Marin, F.; Carbon 1999, 37, 1215.

8. Ladino-Ospina, Y.; Giraldo, L.; Moreno-Piraján, J. C.; J. Therm. Anal. Cal. 2005, 81, 435 .

9. Giraldo, L.; Moreno, J. C.; J. Therm. Anal. Cal. 2007, 89, 589.

10. Radovic, L. R.; Moreno-Castilla, C.; Rivera-Utrilla, J. En Chemistry and Physics of Carbon; Radovic, L. R., ed.; Marcel Dekker: falta cidade, 2001, p. 227.

11. Rodríguez-Reinoso, F. En Introduction to Carbon Technologies; Marsh, H.; Heintz, E. A.; Rodríguez-Reinoso, F., eds.; University of Alicante: falta cidade, 1997, p. 35. 
12. López-Ramón, M. V.; Stoeckli, F.; Moreno-Castilla, C.; Carrasco-Marín, F.; Carbon 2000, 38, 825.

13. Giraldo, L.; Moreno, J. C.; Huertas, J. I.; Inst. Sci. Technol. 2002, 30, 177.

14. Boehm, H. P. En Advances in Catalysis; Eley, D. D. H.; Weisz, P. B., eds.; Academic Press: New York, p. 192.

15. Diaz, C. M.; Briceño, N.; Baquero, M. C.; Giraldo, L.; Moreno, J. C., Internet J. Chem. 2003, 6, GMT 13:52:00.

16. Moreno-Castilla, C.; Rivera-Utrilla, J.; López-Ramón, M. V.; CarrascoMarín, F.; Carbon 1995, 33, 845.
17. Turmuzi, M.; Daud, W. R.; Tasirin, S. M.; Takriff, M. S.; Iyeke, S. E.; Carbon 2004, 42, 453.

18. Wadsö, I.; Wadsö, L., J. Therm. Anal. Cal. 2005, 82, 553.

19. Silvestre-Albero, J.; Sepúlveda-Escribano, C. A.; Rodríguez-Reinoso, F., Colloids Surf. A 2001, 187, 151.

20. Merzougui, Z.; Addoun, F. ; Desalination 2008, 222, 394.

21. Villacañas, F.; Pereira, M. F.; Órfão, J. M.; Figueiredo, J. L., J. Colloid Interface Sci. 2006, 293, 128. 


\section{CARACTERIZACIÓN DEL PROCESO DE ADSORCIÓN DE 3-CLORO FENOL DESDE SOLUCION ACUOSA SOBRE CARBON ACTIVADO POR CALORIMETRIA DE INMERSIÓN}

\section{Liliana Giraldo}

Departamento de Química, Facultad de Ciencias, Universidad Nacional de Colombia, Carrera 30 No 45-03, Bogotá, Colombia Juan Carlos Moreno*

Departamento de Química, Facultad de Ciencias, Universidad de los Andes, Carrera 1 No 18 A-10, Bogotá, Colombia

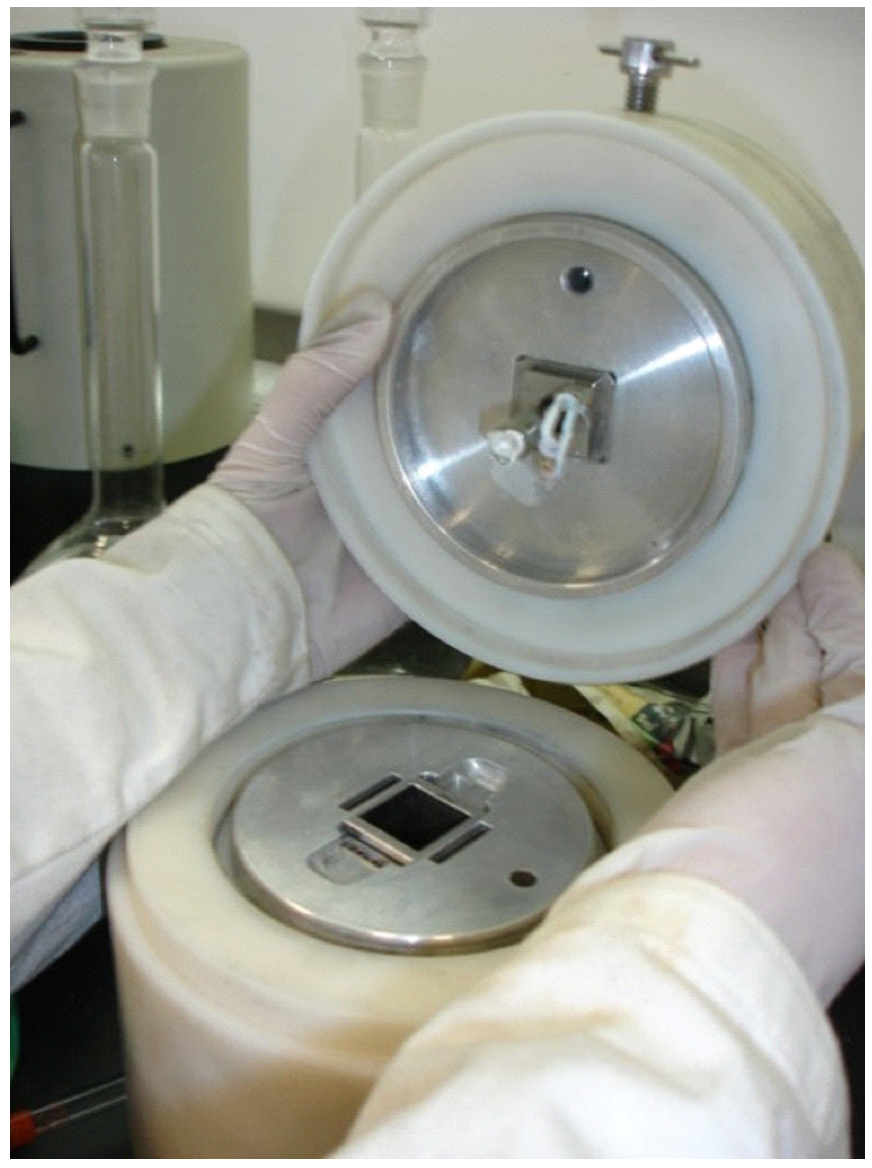

Figura 1S. Fotografía del calorímetro de conducción de calor que se usa en la determinación de las entalpías de inmersión del carbón activado en solución de $100 \mathrm{mgL}^{-1}$ de 3-cloro fenol a $298 \mathrm{~K}$ 